\title{
Inhibition of Histone Deacetylase 6 by Tubastatin A Attenuates the Progress of Osteoarthritis via Improving Mitochondrial Function
}

Yijing Zheng, ${ }^{*}$ Yuemiao Chen, ${ }^{\dagger}$ Xiaolang Lu, ${ }^{*}$ Qihao Weng, ${ }^{*}$ Gaole Dai, ${ }^{*}$ Yang $Y u,{ }^{*}$ Kehe Yu, and Weiyang Gao

From the Departments of Orthopedics* and Hand and Plastic Surgery, ${ }^{\ddagger}$ The Second Affiliated Hospital and Yuying Children’s Hospital of Wenzhou Medical University, Wenzhou, Zhejiang; and the Department of Hematology, ${ }^{\dagger}$ Wenzhou Central Hospital, Wenzhou, Zhejiang, China

Accepted for publication

August 31, 2020.

Address correspondence to Weiyang Gao, B.S., Department of Hand and Plastic Surgery, The Second Affiliated Hospital and Yuying Children's Hospital of Wenzhou Medical University, 109 Western Xueyuan Rd., Wenzhou, Zhejiang 325027, PR China. Email: gaoweiyangwmu@163. com.

\begin{abstract}
Because chondrocytes are the only resident cells in articular cartilage, the steady state of these cells is important for the maintenance of joint function. In various osteoarthritis diseases, chondrocytes undergo a series of pathophysiologic changes, leading to the loss of chondrocytes and the degradation of extracellular matrix (ECM). This study found that Cytoplasmic localized histone deacetylase 6 (HDAC6) is up-regulated on the articular surface in a destabilization of the medial meniscus-induced mouse osteoarthritis model. Because HDAC6 is highly related to the acetylation of tubulin and the function of the microtubule system is closely related to material transport and signal transduction, the relationship between the expression level or activity of HDAC6 and the fate of chondrocytes in vitro and in vivo were confirmed. Primary chondrocytes overexpressing DNA-HDAC6 with plasmid were constructed in vitro, and HDAC6 inhibitor Tubastatin A was selected to inhibit HDAC6 enzyme activity in vivo and in vitro. Subsequently, mitochondrial spatial arrangement, degradation of ECM, and pathological changes in joint were defined. The results indicate that overexpression of HDAC6 causes mitochondrial dysfunction and promotes reactive oxygen species production, leading to degradation of ECM. Tubastatin A treatment after osteoarthritis ameliorates the degradation of cartilage and improves the microenvironment and function of the joint. HDAC6 may be targeted to treat osteoarthritis. (Am J Pathol 2020, 190: 2376-2386; https://doi.org/10.1016/j.ajpath.2020.08.013)
\end{abstract}

Osteoarthritis is a common degenerative joint disease characterized by joint pain and movement disorders. With the aging and obesity of the population, this symptom is more prevalent than in the past few decades. ${ }^{1-3}$ Articular cartilage is a highly specialized tissue composed of only one cell type, chondrocytes, which function in dissipating applied forces and lubricate joint movement. ${ }^{4}$ During the progression of osteoarthritis, chondrocytes undergo a series of pathophysiologic phenomena, such as inflammation, oxidative stress, and apoptosis, which may lead to a reduction in cell density, abnormal secretory activity, and degradation of the extracellular matrix (ECM) to further promote the process of osteoarthritis. ${ }^{5-7}$ Therefore, maintaining the cellular homeostasis of chondrocytes in patients with osteoarthritis may be an effective therapeutic strategy.

Cytoskeleton is a cellular structure that orchestrates cellular events and is predominantly composed of actin microfilaments, tubulin microtubules, vimentin, and nuclear lamin intermediate filaments. ${ }^{8}$ Among these elements, the microtubule system, which is mainly composed of tubulin, plays an important role in maintaining cell morphology, cell division, signal transduction, and material transportation. Previous studies in several cell types, including fibroblasts, ${ }^{9,10}$ synoviocytes, ${ }^{11}$ and fetal chondrocytes, ${ }^{12,13}$ have found that in vitro drug-induced disruption of the microtubule system exhibits a reduction in the synthesis and secretion of collagen and proteoglycan, suggesting that the regulation of the microtubule system may serve as a potential strategy for curing osteoarthritis.

Supported by Fund of Zhejiang Provincial Natural Science Foundation research grant LY18H060011 (Y.Y.) and Public Projects of Zhejiang Province research grant LGD19H060001 (K.Y.).

Disclosures: None declared. 
The function of the microtubule system is mainly regulated by posttranslational modifications of tubulin, such as acetylation, tyrosination, polyglutamylation, and polyglycylation. ${ }^{14,15}$ According to a previous study, acetylation of tubulin is implicated in regulating the stability and function of the microtubule system and thus functions in a variety of cellular activities. ${ }^{16}$ As a member of the histone deacetylase family, histone deacetylase 6 (HDAC6) does not bind to histones like other HDACs do but selectively binds to $\alpha$-tubulin and other substrates in the cytoplasm. ${ }^{16,17}$ Studies on the overexpression of HDAC6 in macrophages and epithelial cells found that the overexpression of HDAC6 significantly induced the production of reactive oxygen species (ROS) and inflammatory cascade through the upregulation of NADPH oxidase expression and activity. ${ }^{18,19}$ Recent studies found that the selective HDAC6 inhibitor ricolinostat (ACY-1215) not only inhibits expression of matrix-degrading proteases, including matrix metallopeptidase (MMP)-1 and MMP-13 in chondrocytes, but also prevents aberrant subchondral bone formation through down-regulating vascular endothelial growth factor expression in osteoblasts. ${ }^{20,21}$ Nevertheless, in view of the offtarget effect and multitarget effect of inhibitors, whether HDAC6 interferes with the generation of ROS and participates in the disease deterioration during the progression of osteoarthritis remains to be further explored.

This study focuses on the variation of HDAC6 expression level in chondrocytes in a mouse model of destabilization of the medial meniscus (DMM). Subsequently, plasmidloading DNA-HDAC6 or the optimized HDAC6 inhibitor Tubastatin A was applied to overexpress HDAC6 or block HDAC6 activity to observe the changes in the fate of the chondrocytes. Finally, the mice were injected intraperitoneally with Tubastatin A to confirm its effect on progression of osteoarthritis, aiming to confirm HDAC6 as a potential therapeutic target for osteoarthritis.

\section{Methods and Materials}

\section{Animals and Ethic Statement}

A total of 48 healthy 8 -week-old, male, C57BL/6OlaCnc mice (20 to $25 \mathrm{~g}$ ) were used for experiments. Sample size was not determined statistically before experimentation. Samples were not randomized. No criteria were used to include or exclude date points or participants. The animals were housed in the OptiMICE Rotary Experimental Animal Cage System (catalog number C89100; Animal Care Systems Inc., Centennial, CO) under standard conditions, including adequate temperature and humidity $(60 \%)$ and a 12-hour light/12-hour dark cycle. The mice had free access to water and food. All experimental procedures were approved by the Laboratory Animal Ethics Committee of Wenzhou Medical University and were performed in accordance with the NIH's Guide for the Care and Use of Laboratory Animals. ${ }^{22}$

\section{Reagents and Antibodies}

Anti-HDAC6 antibody (catalog number 12834-1-AP) and antiglyceraldehyde-3-phosphate dehydrogenase antibody (catalog number 10494-1-AP) were purchased from Proteintech (Chicago, IL). Anti-acetyl- $\alpha$-tubulin (catalog number ab24610), collagen II (catalog number ab34712), MMP-13 (catalog number ab39102), aggrecan (catalog number ab3773), and TOMM20 (catalog number ab186735) were purchased from Abcam (Cambridgeshire, UK). Alexa Fluor 488-labeled and Alexa Fluor 647-labeled goat anti-rabbit/mouse secondary antibodies were purchased from Abcam. Goat anti-rabbit/mouse IgG (heavy chains + light chains) horseradish peroxidase was purchased from MultiSciences (Hangzhou, Zhejiang, China). Primary antibody and secondary antibody removal solution (catalog number P0025) was purchased from Beyotime (Shanghai, China). DAPI-containing anti-fluorescence quenching reagent (catalog number 36308ES20) was purchased from Yeasen (Shanghai, China). Cell Counting Kit 8 solution (catalog number CK04) was purchased from Dojindo Laboratories (Kumamoto Prefecture, Kyushu, Japan). 2'-7'Dichlorofluorescin diacetate probe was purchased from Beyotime (Shanghai, China).

\section{Primary Mouse Chondrocyte Culture}

To obtain primary chondrocytes, knee cartilage tissue of 2 -week-old mice was first dissected. Subsequently, the cartilage was cut into small pieces with sterile scissors and incubated with Dulbecco's modified Eagle's medium/F-12 medium (catalog number 11330032; Gibco, Carlsbad, CA) containing $2 \mathrm{mg} / \mathrm{mL}$ of collagenase II (catalog number $40508 \mathrm{ES} 60$; Yeasen) at $37^{\circ} \mathrm{C}$ for 4 hours. After washing and resuspending, the obtained chondrocytes were inoculated in Dulbecco's modified Eagle's medium/F-12 medium supplemented with $10 \%$ fetal bovine serum (catalog number p30-3301; PAN-Biotech GmbH, Adenbach, Baghlia, Germany), 1\% penicillin/streptomycin (catalog number 15140122; Gibco). The cells were cultured in an incubator with $5 \%$ carbon dioxide and a temperature of $37^{\circ} \mathrm{C}$ and replaced with fresh culture medium after 24 hours of culture. The cells were digested with $0.25 \%$ trypsin-EDTA (catalog number 12605028; Gibco) and seeded in new culture dishes when the cell confluence reached $80 \%$. The primary chondrocytes after the second generation were used in related experiments. To confirm the toxicity of Tubastatin A, $1 \times 10^{4}$ cells were seeded in 96-well plates per well for 48 hours, and then cellular viability was measured with the Cell Counting Kit 8 according to its manual after 1 hour of Tubastatin A treatment. As for immunofluorescence and Western blot (WB) analysis, $1 \times 10^{5}$ cells and $2 \times 10^{5}$ cells were seeded in 12-well plates (containing sterile slides) and 6-well plates per well for 48 hours, respectively.

\section{Plasmid Transfection}

DNA-HDAC6 (plasmid GV230) and empty vector (plasmid GV230) were purchased from Genechem 
(Shanghai, China). According to the instructions, DNAHDAC6 was transfected into primary chondrocytes in the presence of Lipo3000 (catalog number L3000001; ThermoFisher, Waltham, MA). Inverted fluorescence microscope was used to image the intracellular enhanced green fluorescent protein 48 hours after transfection to determine the efficiency of plasmid transfection. The cells were used in subsequent experiments after the transfection efficiency was confirmed as $>50 \%$

\section{Animal Model}

A mouse osteoarthritis model was induced by surgical DMM as previously described. ${ }^{23}$ The mice in the sham group was randomly selected and received arthrotomy in the left knee joint without removing the medial meniscotibial ligament. After the operation, the mice in the osteoarthritis group were randomly divided into two groups with or without Tubastatin A treatment $(50 \mathrm{mg} / \mathrm{kg}$ daily i.p., catalog number HY-13271A; MedChemExpress, Monmouth Junction, NJ), so that all mice were randomly divided into a sham group, osteoarthritis group, and Tubastatin A-treated osteoarthritis group. Unless otherwise stated, mice were sacrificed 8 weeks after surgery.

\section{Evaluation of Joint Hyperalgesia and Joint Swelling}

In view of the significance of the knee-bend test in evaluating dynamic hyperalgesia in rat joints, a similar evaluation system on mice was performed to evaluate joint hyperalgesia as previously described. ${ }^{24}$ Before DMM surgery, evaluation of joint hyperalgesia was performed as a baseline and then every 4 days until day 28. Each group contains 6 mice, and each mouse was evaluated three times to ensure accuracy. After the evaluation on the 28th day after DMM surgery, the measurement of the bilateral knee diameter was conducted with a calibrated digital caliper (the measurement was repeated three times to obtain a mean value), and then the absolute value of the difference in the bilateral knee diameter was calculated to evaluate the degree of joint swelling. The entire process was performed by professional investigators in a blind manner (Y.Y. and K.Y.).

\section{Quantitation of Glycosaminoglycan and ROS}

At 4 weeks after surgery, mice were sacrificed by i.p. injection of excess $4 \%$ chloral hydrate. The knee joints of mice were isolated and rinsed three times with sterile saline, and then the joint cavity was exposed and quickly rinsed twice with a total of $100 \mu \mathrm{L}$ of sterile normal saline containing $4 \mathrm{mmol} / \mathrm{L}$ EDTA (catalog number E8030; Solarbio, Beijing, China). The synovial membrane lavage fluid was centrifuged at $4^{\circ} \mathrm{C}$ for 10 minutes at $850 \times g$, and the supernatant was collected for subsequent detection. After synovial fluid was collected, the cartilage tissue was carefully obtained with a scalpel. Subsequently, under the guidance of the instructions, the dihydroethidium (DHE)ROS detection kit (catalog number BB-47051-1; BestBio, Shanghai, China) was used to detect the level of ROS in the joint cavity, and the mouse glycosaminoglycan enzyme-linked immunosorbent assay kit (catalog number ml063815; Mlbio, Shanghai, China) was used to measure the content of glycosaminoglycan in articular cartilage. The intensity of DHE-ROS was measured by a synergy H1 hybrid multidetector microplate reader (BioTek, Winooski, VT). The processes of the DHE-ROS detection kit and the mouse glycosaminoglycan enzyme-linked immunosorbent assay kit were conducted by professional investigators in a blind manner (X.L. and Q.W.). The samples in each group were derived from six independent mice, and triplicate wells were set to ensure accuracy.

\section{WB Analysis}

Primary chondrocytes were lysed in lysis buffer (catalog number AR0103; Boster, Wuhan, Hubei, China) supplemented with protease inhibitors (catalog number P1005; Beyotime, Shanghai, China) To obtain total cell protein, followed by centrifugation at $13,680 \times g$ for 15 minutes at $4^{\circ} \mathrm{C}$. Bradford (catalog number ab119216; Abcam) was then used to quantify the protein concentrations in cell lysate supernatant. Equal amounts of protein samples from each group were separated by electrophoresis on $10 \%$ to $12 \%$ SDS-PAGE gels in the presence of loading buffer (catalog number P0015L; Beyotime). The proteins were then transferred onto a polyvinylidene fluoride membrane (catalog number 1620256; Bio-Rad, Berkeley, CA) and blocked in 5\% skim milk at room temperature for 1.5 hours, followed by incubated with designated primary antibodies overnight at $4^{\circ} \mathrm{C}$. The next day, the polyvinylidene fluoride membrane was washed with Trisbuffered saline with Tween 20 three times $(7$ minutes each time) and incubated with the secondary antibody at room temperature for 1.5 hours. A ChemiDicTM XRS+ Imaging System (Bio-Rad) was used to visualize the signals on membrane, and the bands were quantified by densitometric measurement in Quantity-One software version 4.6.2 (Bio-Rad). The visualization and quantification were conducted by professional investigators in a blind manner (Y.C., X.L., and G.D.).

\section{Tissue Preparation and Histopathologic Analysis}

At 2, 4, and 8 weeks after surgery, the mice were sacrificed by i.p. injection of excess $4 \%$ chloral hydrate to obtain knee joints. The knee joint was soaked in $4 \%$ paraformaldehyde for 24 hours and then decalcified in $10 \%$ EDTA solution for 4 weeks. The knee joint was then dehydrated by gradient dehydration and embedded in paraffin, followed by cutting into $5-\mu \mathrm{m}$ sagittal sections. Sections were stained with safranin $\mathrm{O}$-fast green (catalog number G1371; Solarbio) and hematoxylin and eosin 


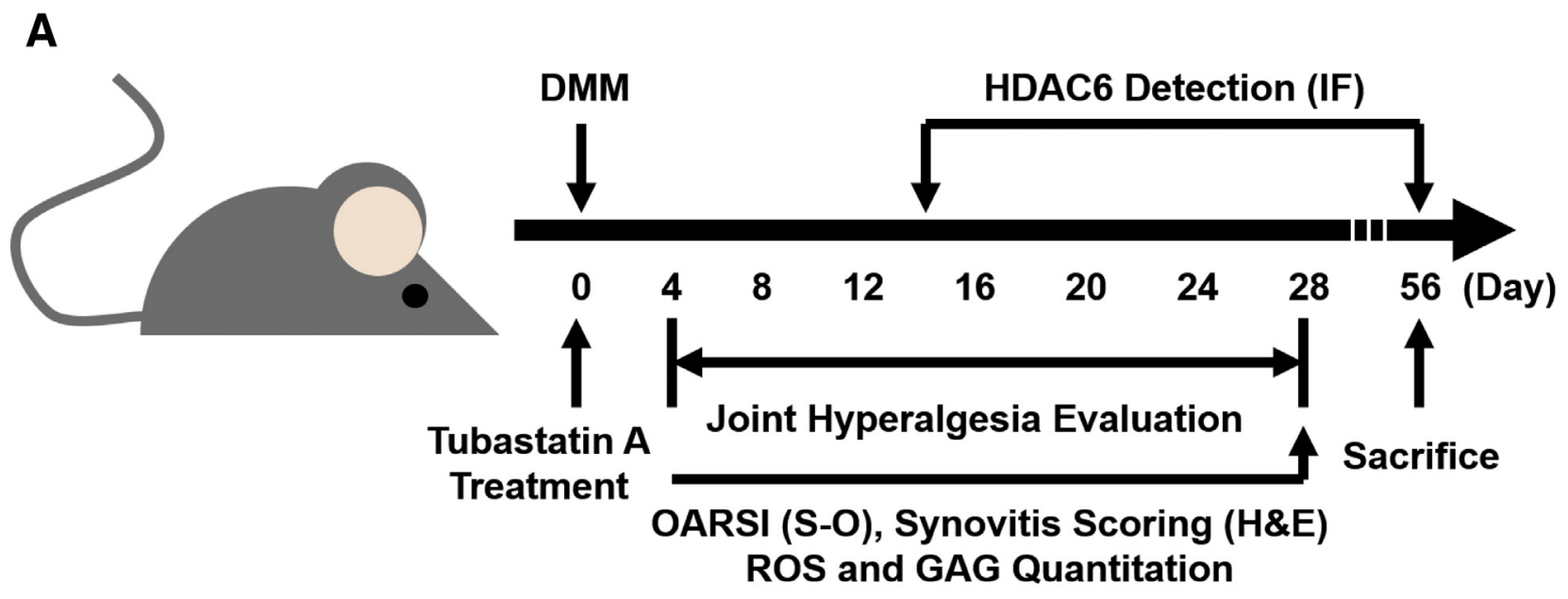

B
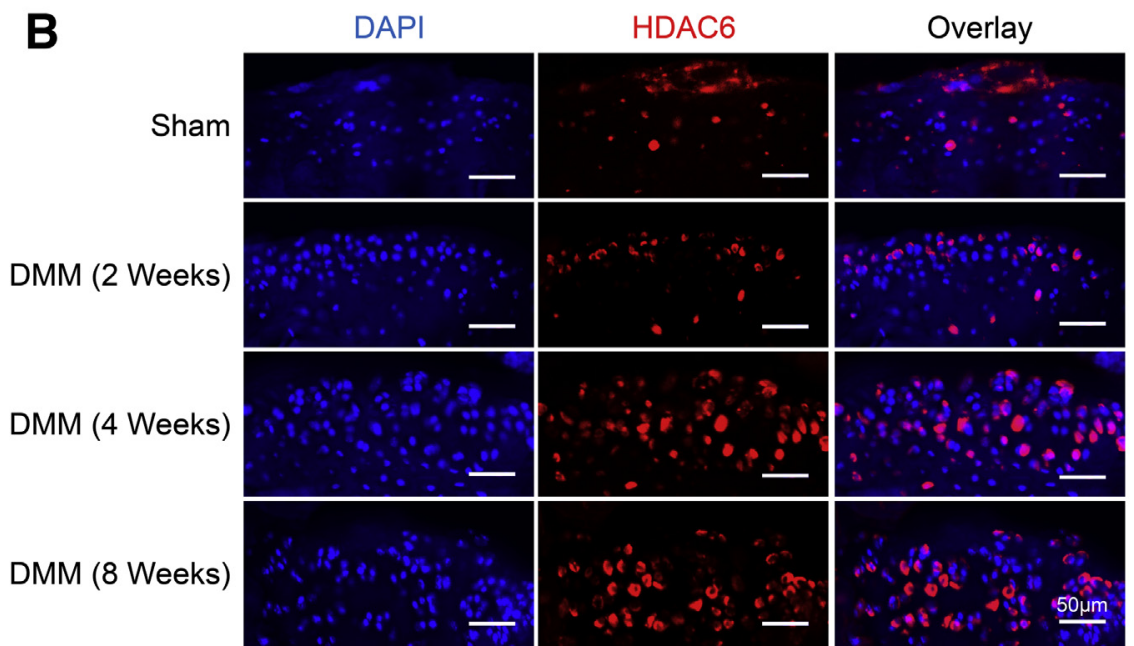

C

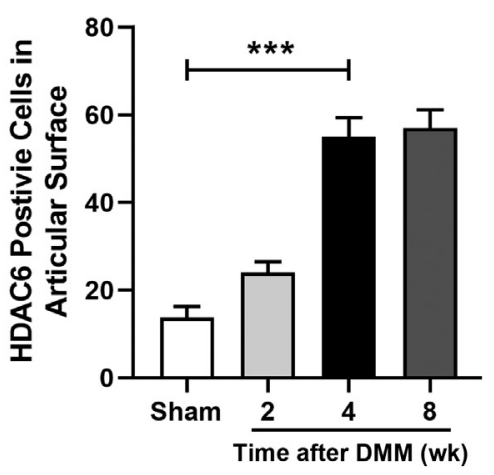

Figure 1 The schedule of experiments and expression level of histone deacetylase 6 (HDAC6) in a destabilization of the medial meniscus (DMM)-induced mouse osteoarthritis model. A: Schedule for drug treatment, model construction, and experimental analysis. B and C: Representative images and quantitative analysis of HDAC6-positive chondrocytes in cartilage. Data are expressed as means \pm SEM. $n=3$ independent mice. ${ }^{* *} P<0.001$ versus sham. Scale bars $=50 \mu \mathrm{m}(\mathbf{B})$. GAG, glycosaminoglycans; H\&E, hematoxylin and eosin; IF, immunofluorescence; ROS, reactive oxygen species; S-0, safranin 0-fast green.

(catalog number G1120; Solarbio) according to guidance of their instruction to observe histopathologic changes. The cellularity and morphology of cartilage and subchondral bone cells were observed by the experimenter under a microscope in a blinded manner (Y.C., Y.Y., and K.Y.), and the medial femoral condyle and medial tibial plateaus were scored according to the International Association for Osteoarthritis Research (OARSI) scoring system. The scoring system on which the severity of synovitis was evaluated has been previously described. ${ }^{25}$ The entire experiments were conducted by professional investigators in a blind manner (Y.C., Y.Y., and K.Y.).

\section{Immunofluorescence Analysis}

The previously described tissue sections (Tissue Preparation and Histopathologic Analysis) were dewaxed, gradient hydrated, and high-pressure antigen repaired, followed by incubating with phosphate-buffered saline solution (catalog number 10010023; Gibco) containing $0.2 \%$ Triton X-100 (catalog number T8200; Solarbio) and $5 \%$ fetal bovine serum at room temperature for 30 minutes to block nonspecific binding sites. The sections were then incubated with secondary antibodies at room temperature for 1 hour after incubating overnight with designated primary antibodies at $4{ }^{\circ} \mathrm{C}$. DAPI-containing antifluorescence quenching reagent was used to stain the nucleus and mount. Images of the same marker between different groups were captured by a Nikon ECLIPSE 80i microscope (Nikon, Tokyo, Japan) at the same laser intensity. The capture and images analyses were conducted by professional investigators in a blind manner (Y.Z., Y.C., and X.L.).

\section{Statistical Analysis}

All data were expressed as the means \pm SEM from three independent experiments. In addition to joint hyperalgesia and swelling assessment (six mice per group), 

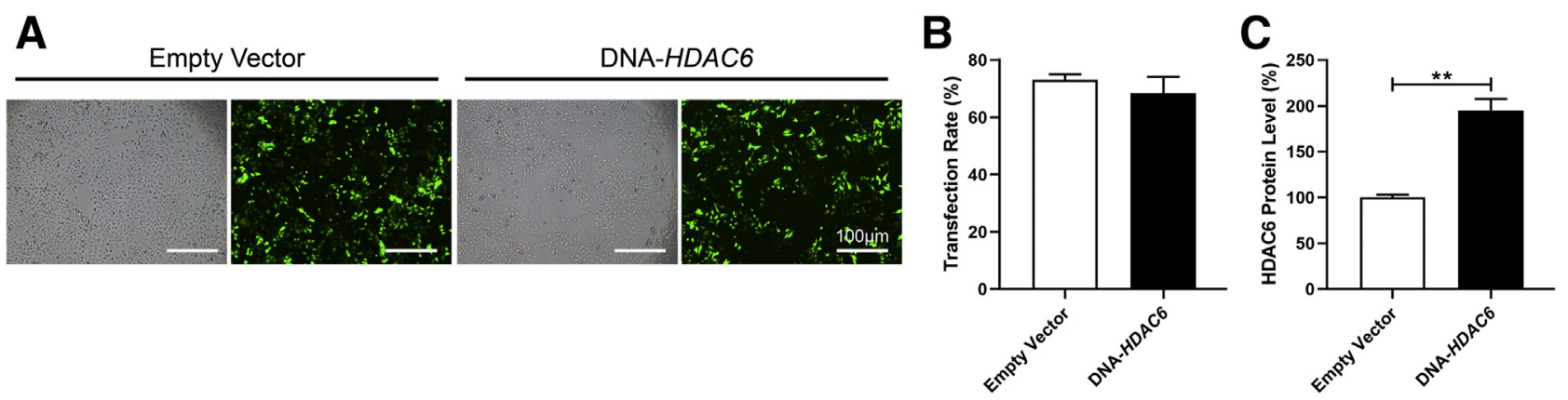

D

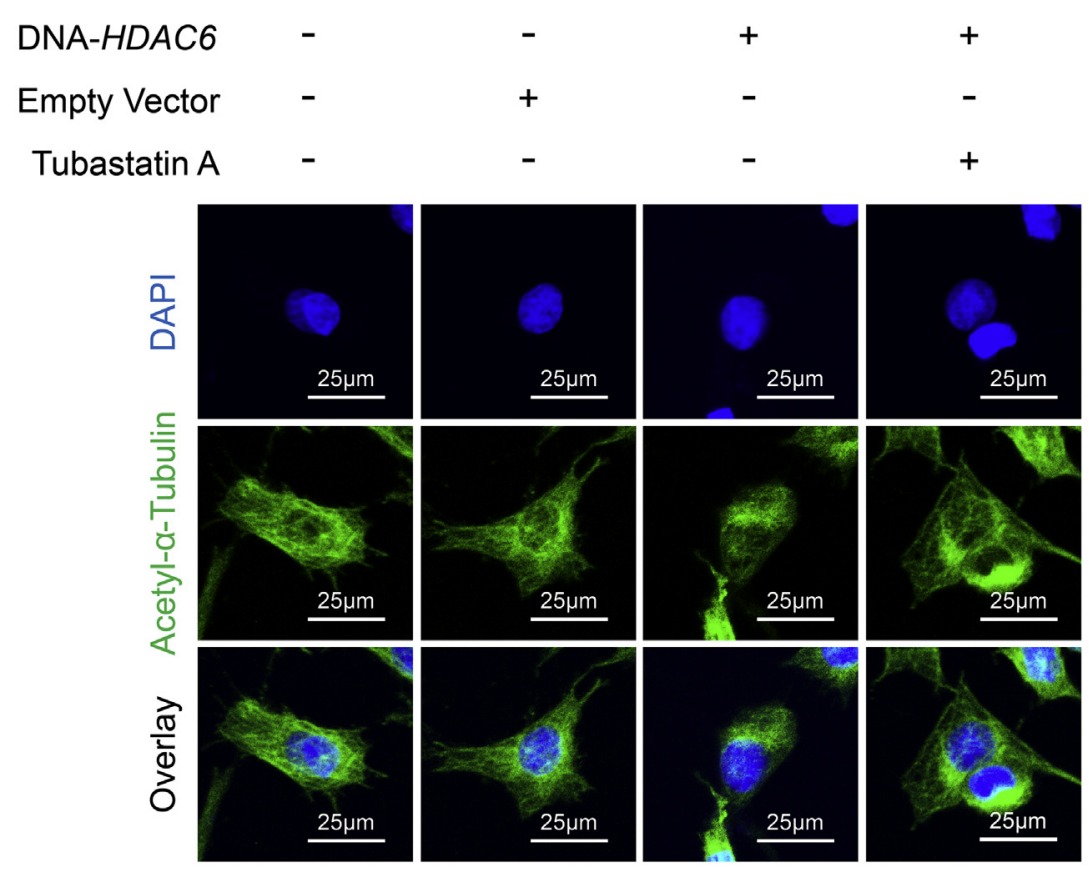

G

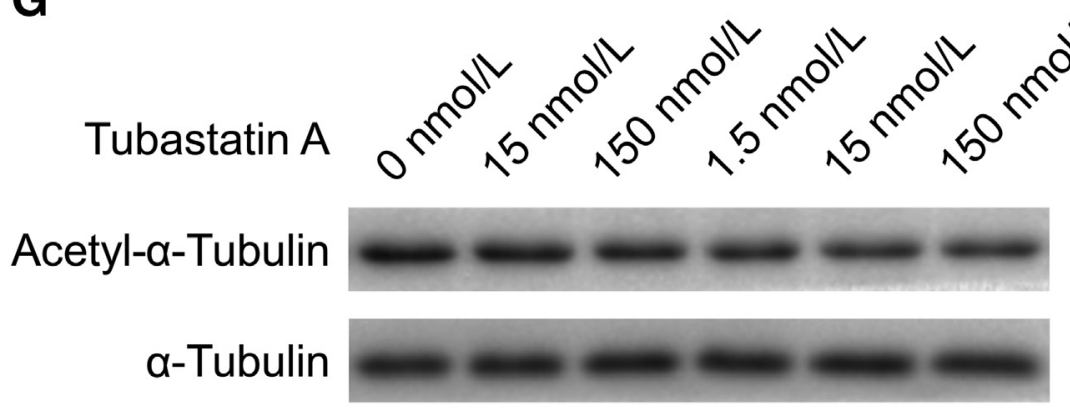

E

\section{HDAC6}

GAPDH
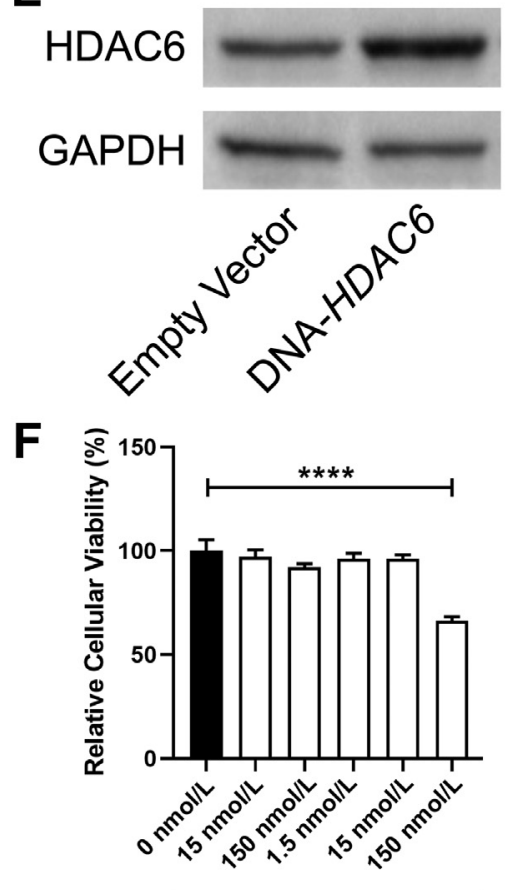

Tubastatin A

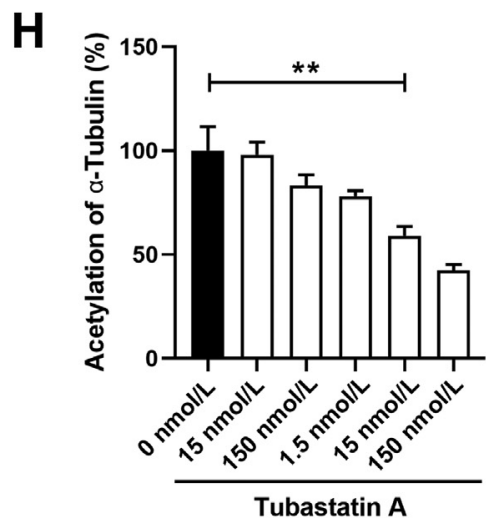

Figure 2 In vitro construction of primary chondrocytes overexpressing histone deacetylase 6 (HDAC6). A and B: Representative images and quantitative analysis of primary chondrocytes transfected with plasmid. C and E: Western blot analysis and quantitative analysis of expression level of HDAC6 in cells. D: Representative images of cells stained with anti-acetyl- $\alpha$-tubulin antibody. F: Quantitative analysis of cellular viability. $\mathbf{G}$ and $\mathbf{H}$ : Western blot analysis and quantitative analysis of acetylation of tubulin in cells. Data are expressed as means \pm SEM. $n=3$ independent wells $(\mathbf{A}-\mathbf{E}$ and $\mathbf{G}$ and $\mathbf{H}) ; n=6$ independent wells (F). ${ }^{* *} P<0.01,{ }^{* * * P}<0.0001$. Scale bars: $100 \mu \mathrm{m}$ (A); $25 \mu \mathrm{m}$ (D). GAPDH, glyceraldehyde-3-phosphate dehydrogenase.

glycosaminoglycan and ROS content determination (six mice per group), and cellular viability analysis, the remaining experiments in each group contained three independent mice or three independent wells (for cells). The statistical significance between two groups and multiple groups were respectively analyzed by the $t$-test and one-way analysis of variance test, followed by Tukey multiple comparison test. Nonparametric data (OARSI scores and 

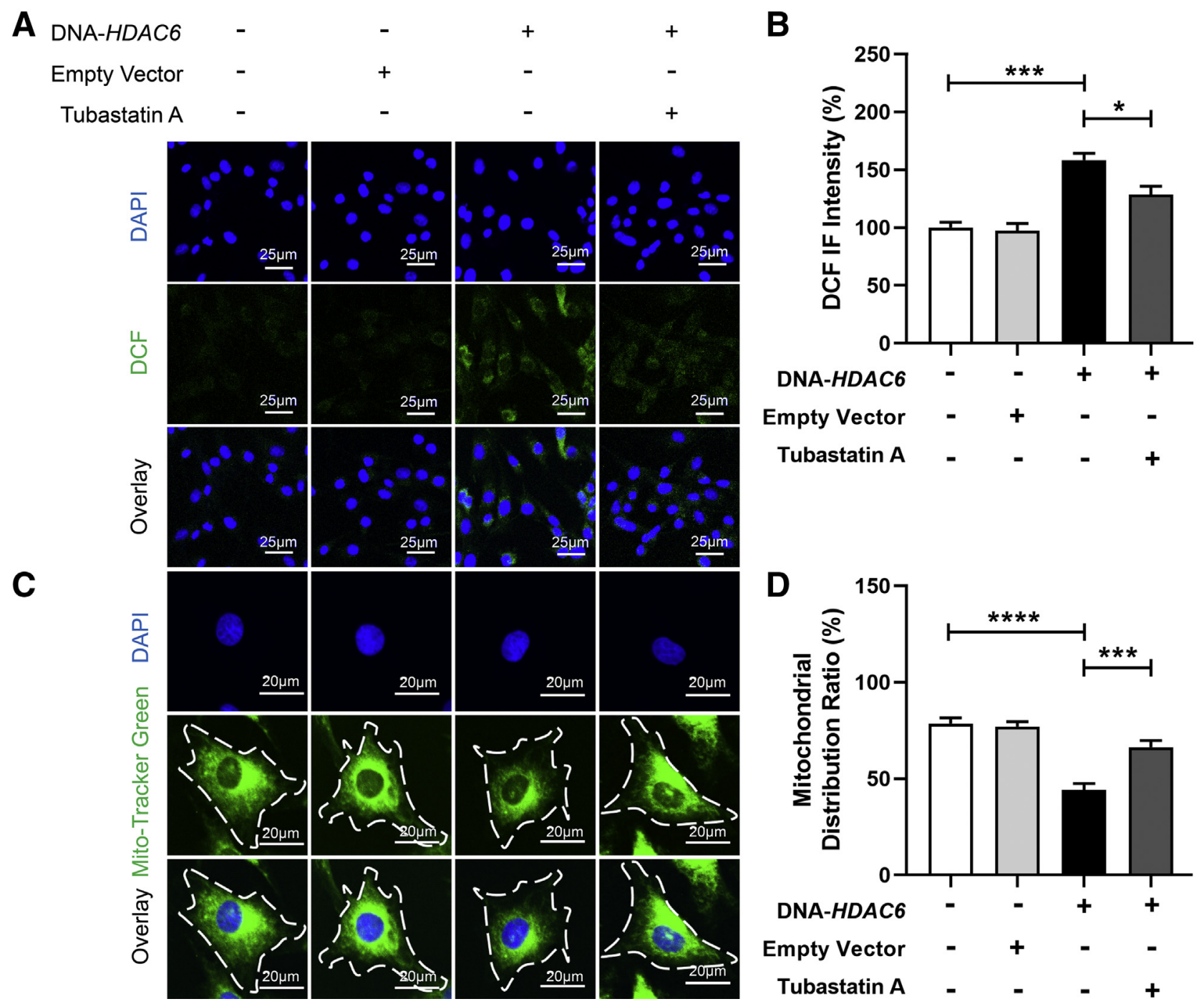

Figure 3 The significance of histone deacetylase 6 (HDAC6) expression in primary chondrocytes. A and B: Representative images of cells stained with 2'-7'dichlorofluorescin (DCF) probe and quantitative analysis of DCF intensity in cells. C and D: Representative images of cells stained with Mito-Tracker Green probe and analysis of proportion of Mito-Tracker Green-positive area to total cellular area. White dotted lines depict the outline of the cell. The cells were treated with Tubastatin A at a concentration of $15 \mu \mathrm{mol} / \mathrm{L}(\mathbf{A}-\mathbf{D})$. Data are expressed as as means \pm SEM. $n=3$ independent wells. ${ }^{*} P<0.05,{ }^{* * *} P<0.001$, and $* * * P<0.0001$. Scale bars: $25 \mu \mathrm{m}(\mathbf{A}) ; 20 \mu \mathrm{m}(\mathbf{C})$.

synovitis scores) were analyzed by the Kruskal-Wallis $\mathrm{H}$ test, followed by Tukey multiple comparison test. $P<0.05$ was considered statistically significant.

\section{Results}

Variation of Expression Level of HDAC6 in Progression of DMM-Induced Mouse Osteoarthritis Model

The time schedule in Figure 1A shows the process and methods of research. To determine the variation of expression level of HDAC6 after osteoarthritis, immunofluorescence staining with anti-HDAC6 antibody on the joints of mice 2, 4, and 8 weeks after DMM surgery were conducted and revealed that the expression level of
HDAC6 was increased with the development of osteoarthritis and peaked at the fourth week in cells of the articular surface (Figure 1, B and C). Considering the role of cartilage on the articular surface, the role of HDAC6 in chondrocytes was explored.

\section{Construction of Primary Chondrocyte Overexpressing HDAC6 in Vitro}

Primary chondrocytes were transfected with a plasmid vector carrying the HDAC6 gene in vitro to overexpress HDAC6. After the transfection was completed, the enhanced green fluorescent protein-positive cells were imaged and showed that at least $70 \%$ of primary chondrocytes were successfully transfected (Figure 2, A and B). WB analysis exhibits that the expression level of HDAC6 was significantly increased in 


\section{A}
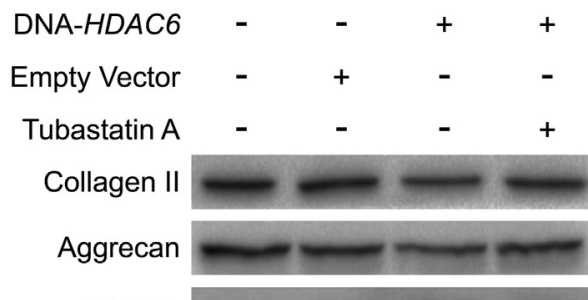

MMP13

TOMM20

GAPDH

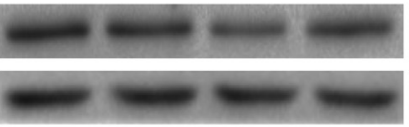

B

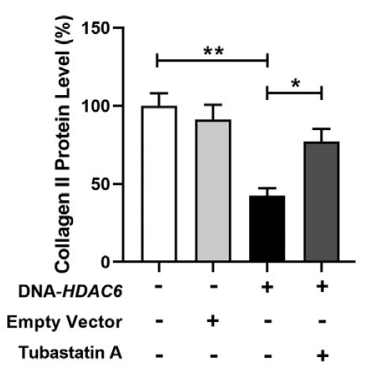

E

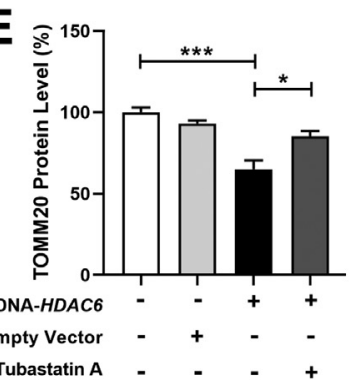

Figure 4 Effect of histone deacetylase 6 (HDAC6) expression on protein expression level. A-E: Western blot analysis and quantitative analysis of expression level of collagen II, aggrecan, matix metalloproteinase (MMP)-13 and TOMM20 in cells. The cells were treated with Tubastatin $A$ at a concentration of $15 \mu \mathrm{mol} / \mathrm{L}$. Data are expressed as means \pm SEM. $n=3$ independent wells. ${ }^{*} P<0.05$ Tubastatin A+DNA-HDAC6 group versus DNA-HDAC6 group (B, C. and E) ${ }^{* *} P<0.01,{ }^{* * *} P<0.001$. primary chondrocytes after plasmid transfection (Figure 2, C and E). In immunofluorescence analysis, compared with the dispersed expression acetyl- $\boldsymbol{\alpha}$-tubulin in the cells of the control group (without transfection) and the vector transfection group, acetyl- $\alpha$-tubulin clusters around the nucleus in the HDAC6 overexpression group (Figure 3D).

\section{Administration of Tubastatin A to Primary Chondrocytes in Vitro}

Optimized HDAC6 inhibitor Tubastatin A was then applied to better define the significance of HDAC6 in primary chondrocyte in vitro. As a highly selective HDAC6
A

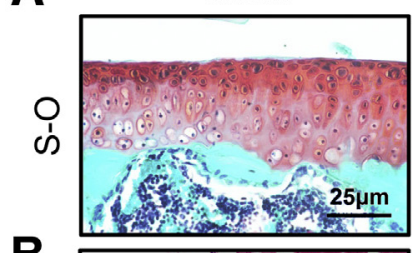

B
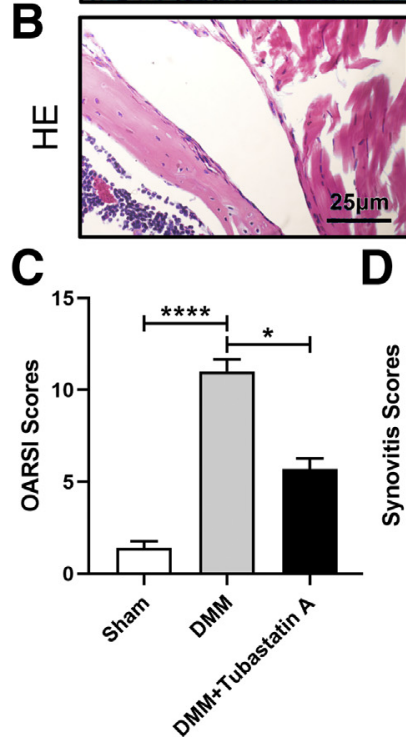

D

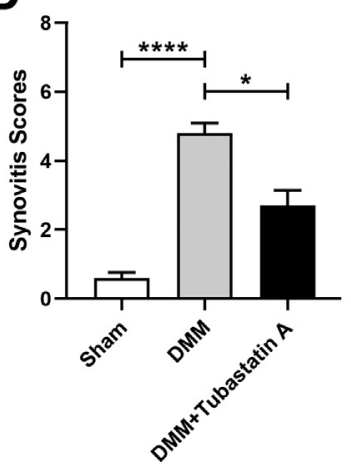

OA+Tubastatin A
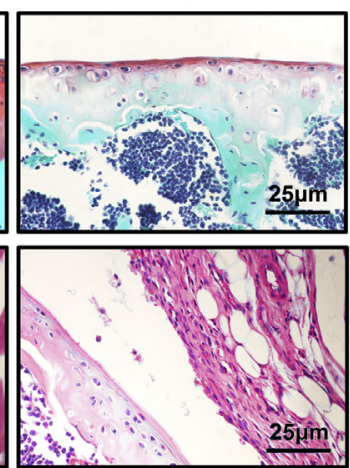

E

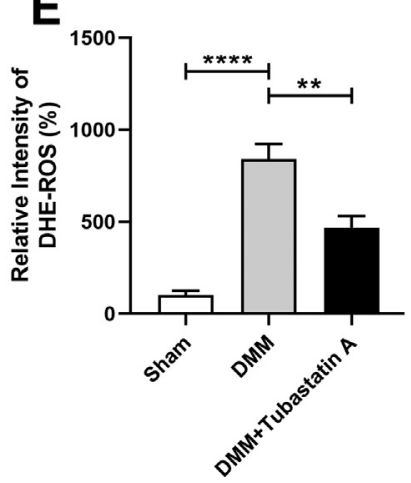

Figure 5 The effect of Tubastatin A treatment on pathology of a destabilization of the medial meniscus (DMM)-induced mouse osteoarthritis $(0 \mathrm{~A})$ model. A: Representative images of safranin 0 -fast green (S-0) staining of mouse articular cartilage. B: Representative images of hematoxylin and eosin (H\&E) staining of mouse articular synovium. C and D: Quantitative analysis of pathology of articular cartilage and articular synovium via the International Association for Osteoarthritis Research (OARSI) and the synovitis scoring systems. E: Quantitative analysis of relative intensity of dihydroethidium (DHE)-reactive oxygen species (ROS) in synovial membrane lavage fluid. Data are expressed as means \pm SEM. $n=6$ independent mice in each group $(\mathbf{C}-\mathbf{E}) .{ }^{*} P<0.05$, $* * P<0.01$, and ${ }^{* * * *} P<0.0001$. Scale bars $=25$ $\mu \mathrm{m}$ (A and $\mathbf{B})$. 

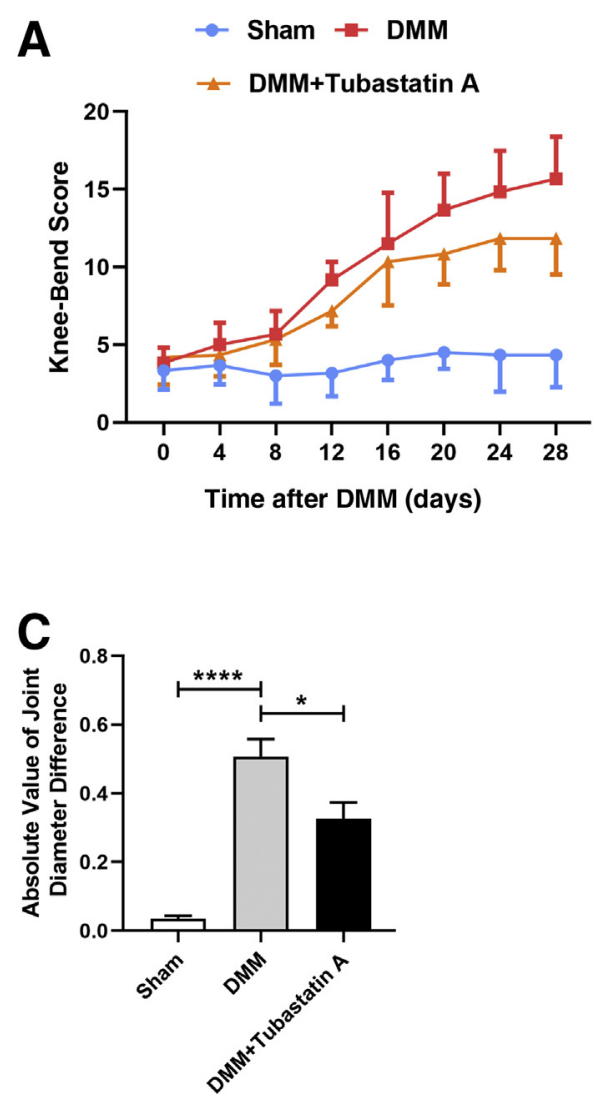

A
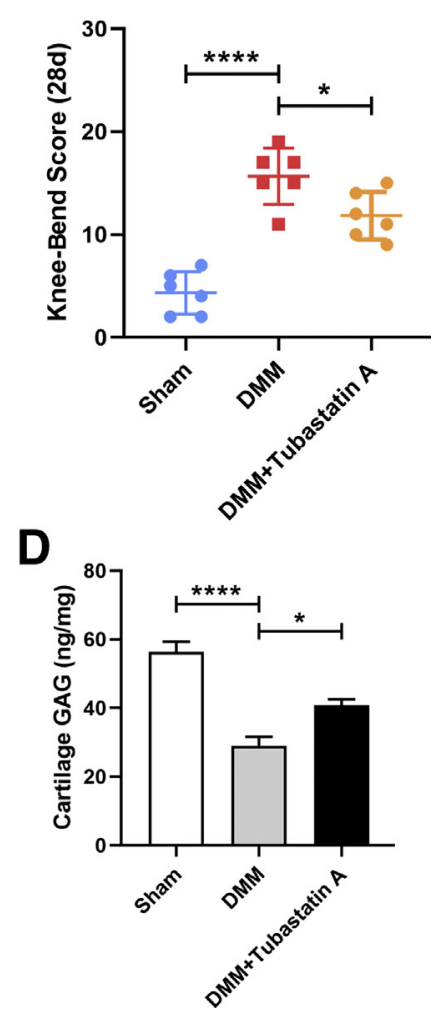

Figure 6 The therapeutic effect of Tubastatin A treatment on a destabilization of the medial meniscus (DMM)-induced mouse osteoarthritis model. A: Analysis of joint hyperalgesia after a DMM-induced mouse osteoarthritis model via knee-bend scoring system. B: Quantitative analysis of joint hyperalgesia at 28 days after DMM. C: Quantitative analysis of absolute value of bilateral knee joint diameter difference. D: Quantitative analysis of glycosaminoglycan content in cartilage of knee joint. Data are expressed as means \pm SEM. $n=6$ independent mice in each group (A-D). ${ }^{*} P<0.05,{ }^{* * *} P<0.0001$. inhibitor, Tubastatin A, has a half maximal inhibitory concentration of $15 \mathrm{nmol} / \mathrm{L}$ for HDAC6 in a cell-free assay and requires a much higher half maximal inhibitory concentration for HDACs (1000-fold more) other than HDAC8 (57fold more). ${ }^{26}$ The results of $\mathrm{WB}$ analysis indicate that the acetylation level of $\alpha$-tubulin decreases in a concentrationdependent manner, and a significant difference appears at $15 \mu \mathrm{mol} / \mathrm{L}$ (Figure 2, $\mathrm{G}$ and $\mathrm{H}$ ). In addition, cellular viability analysis indicates that at $150 \mu \mathrm{mol} / \mathrm{L}$, Tubastatin A exhibits significant cytotoxicity (Figure 2F). Therefore, 15 $\mu \mathrm{mol} / \mathrm{L}$ of Tubastatin A was applied in in vitro experiments. Compared with the HDAC6 overexpression group, the treatment of Tubastatin A rescues the deacetylation of tubulin in primary chondrocyte caused by HDAC6 overexpression and achieved a more regular arrangement of cellular microtubules (Figure 2D) in immunofluorescence staining with anti-acetyl- $\alpha$-tubulin antibody.

\section{Significance of Tubastatin A to Primary Chondrocytes in Vitro}

Given the importance of tubulin acetylation for mitochondrial spatial localization, Mito-Tracker Green was used to identify the mitochondrial morphologic features and distribution in primary chondrocytes overexpressing HDAC6 treated with or without Tubastatin A. The results indicate that the overexpression of HDAC6 caused Mito-Tracker Green-positive mitochondria not to extend to the cell edge like the control group and vehicle transfection group but to gather in the perinuclear area, whereas Tubastatin A treatment significantly increases localization of Mito-Tracker Green-positive spots at the cell edge compared with the HDAC6 overexpression group (Figure 3, C and D). In addition, the results of 2'-7'dichlorofluorescin diacetate probe detection of ROS exhibit that the application of Tubastatin A can rescue the ROS level enhanced by HDAC6 overexpression to a level close to that of the control group and the vector transfection group (Figure 3, A and B).

Protein-level analyses indicate that the overexpression of HDAC6 in primary chondrocytes results in a significant down-regulation of the expression levels of TOMM20, aggrecan, and collagen II and a significant up-regulation of the expression level of MMP-13 compared with the control group and vehicle group, whereas the treatment of Tubastatin A reverses the effect caused by HDAC6 overexpression (Figure 4). The above results reveal the effect of HDAC6 on ECM degradation and mitochondrial arrangement in primary chondrocytes in vitro.

Significance of HDAC6 to Progression of DMM-Induced Mouse Osteoarthritis Model

Tubastatin A were eventually injected into mice intraperitoneally to observe the effect of inhibition of HDAC6 


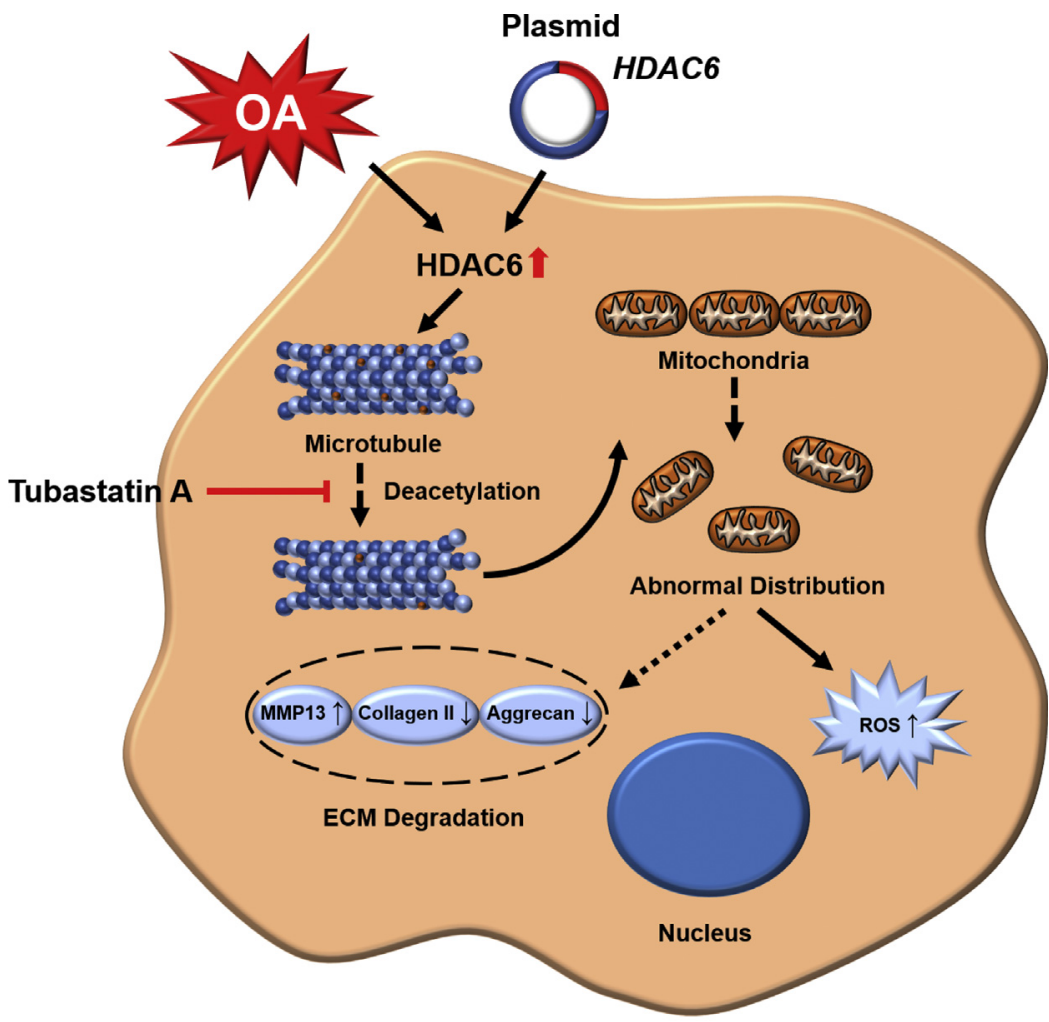

Figure 7 Because the expression level of histone deacetylase 6 (HDAC6) was enhanced after a destabilization of the medial meniscus (DMM)-induced mouse osteoarthritis $(\mathrm{OA})$ model, primary chondrocytes overexpressing DNA-HDAC6 were constructed via plasmid transfection. The results indicate that the up-regulation of HDAC6 can deacetylate tubulin in the microtubule system, which interferes with spatial arrangement of mitochondria, leading to degradation of the extracellular matrix (ECM) and up-regulation of reactive oxygen species (ROS), whereas inhibition of the enzyme activity of HDAC6 by Tubastatin A treatment reversed the results caused by HDAC6 overexpression in vitro and postponed the development of $\mathrm{OA}$ in vivo, suggesting HDAC6 as a promising target for $\mathrm{OA}$ cure. MMP, matrix metalloproteinase.

Chondrocyte

enzyme activity on the DMM-induced osteoarthritis model. Histopathological analyses by safranin $\mathrm{O}$-fast green and hematoxylin and eosin staining prove that the osteoarthritis group exhibits degraded cartilage surface tissue and thickened synovial tissue compared with the sham group, whereas treatment with Tubastatin A to inhibit enzyme activity of HDAC6 obviously inhibits degradation of cartilage surface tissue and synovial tissue thickening (Figure 5, A and B). Consistent with histopathological analyses, evaluations based on the OARSI scoring system and synovitis scoring system also prove that the treatment of Tubastatin A significantly reduced pathologic score after osteoarthritis (Figure 5, C and D).

Knee-bend analysis of the knee hyperalgesia in mice for 4 weeks reveals that DMM induced serious hyperalgesia compared with the sham group, whereas the application of Tubastatin A significantly alleviates hyperalgesia, and the significant difference appears at 4 weeks after DMM surgery (Figure 6, A and B). Analysis of the difference in bilateral knee joint diameters in mice found that treatment with Tubastatin A can significantly reduce DMM-induced joint swelling (Figure 6C). The results of the measurement of the ROS content with a DHE-ROS detection kit in the joint cavity and the glycosaminoglycan content in the cartilage tissue indicate that, compared with the sham group, DMM causes a significant increase in ROS level but decreased glycosaminoglycan content. However, the administration of Tubastatin A in the DMM-induced osteoarthritis model significantly inhibited the level of ROS and increased the concentration of glycosaminoglycan in the joint cavity (Figures 5E and 6D).

\section{Discussion}

Chondrocytes are the only resident cells of articular cartilage, and the steady state of their intracellular environment is of great significance for the maintenance of articular cartilage morphology. This study found that the expression of HDAC6 in cells of the articular surface increased in the DMM-induced mouse osteoarthritis model. HDAC6 is a cytoplasmic localized histone deacetylase that has deacetylase activity and ubiquitinated protein-binding ability. ${ }^{17,27}$ The acetylation of tubulin caused by deacetylase activity of HDAC6 is of great significance for the regulation of the function of the microtubule system.

Tubulin acetylation is widely involved in organelle location, material transport, signal transduction, and cell movement. There is evidence that acetylation of tubulin mediates dynein-dependent transport of mitochondria, thereby affecting the spatial arrangement of 
mitochondria. ${ }^{28,29}$ Lee et $\mathrm{al}^{30}$ proved that ubiquitinated mitochondria can recruit and bind HDAC6 to mediate lysosomal-dependent mitophagy. In addition, a genomewide RNA interference screen in Drosophila cells using mitochondrial citrate synthase activity as the primary readout identified HDAC6 as a regulator of mitochondria function. ${ }^{31}$ However, the effect of HDAC6 on mitochondria in the context of osteoarthritis remains to be investigated.

Owing to the dominant role of chondrocytes in the articular surface, primary chondrocytes overexpressing HDAC6 were transfected with a plasmid vector in vitro. Under the premise of ensuring high transfection efficiency, the expression level of the HDAC6 protein and the degree of acetylation of $\alpha$-tubulin were detected. After overexpressing HDAC6, mitochondria reduced their localization at the cell edge and accumulated around the primary chondrocyte nucleus. The regulation of activity, assembly, distribution, and stability of mitochondria was involved in mitochondrial fission and fusion, thereby mediating cell growth, mitosis, apoptosis, and human diseases. ${ }^{32}$ It was certified that mitochondrial dysfunction, including variation in mitochondrial respiratory chain activity or ATP synthesis, is implicated in the development of osteoarthritis and may affect cartilage degradation. ${ }^{33,34}$ Nevertheless, there is currently a lack of research on the spatial distribution of mitochondria in the context of osteoarthritis. Whether mitochondrial dysfunction after osteoarthritis includes mitochondrial distribution disorders remains to be investigated.

To better define the role of HDAC6 in chondrocytes after osteoarthritis, the HDAC6 inhibitor Tubastatin A was selected to interfere with the enzyme activity of HDAC6. The in vitro results indicate that the treatment of primary chondrocytes overexpressing HDAC6 with Tubastatin A significantly promoted the acetylation of $\alpha$-tubulin, increased the number of mitochondria (increased expression level of TOMM20), improved the spatial location of mitochondria, and inhibited ROS production. Because numerous preclinical studies have confirmed that improving mitochondrial function can alleviate degradation of cartilage after osteoarthritis, ${ }^{35,36}$ WB analysis was conducted and found that the overexpression of HDAC6 induced the degradation of ECM (decrease of collagen II and aggrecan and increase of MMP-13), whereas the administration of Tubastatin A reversed the degradation.

In addition, in vivo experiments proved that inhibition of HDAC6 activity by i.p. injection of Tubastatin A diluted with saline in the DMM-induced mouse osteoarthritis model can inhibit cartilage degradation, reduce occurrence of synovitis, and improve joint hyperalgesia and swelling. Because studies have found that intra-articular injection of monoiodoacetate-induced osteoarthritis model mice receiving saline treatment has no effect on the progression of osteoarthritis, ${ }^{37}$ the additional vehicle group is not added. To better quantify the degradation of cartilage, the matrix protein glycosaminoglycan in cartilage was measured to confirm the inhibitory effect of Tubastatin A on the degradation of the ECM in the osteoarthritis process. In view of the close relationship between mitochondrial dysfunction and ROS production and the critical role of ROS in the development of osteoarthritis, ${ }^{38}$ the concentration of ROS was measured by DHE-ROS detection kit in synovial membrane lavage fluid and found that Tubastatin A treatment after DMM surgery significantly alleviated ROS production. These results suggest that HDAC6 may affect the mitochondrial spatial arrangement to resist mitochondrial dysfunction, thus mediating the degradation of cartilage and deterioration of the microenvironment after osteoarthritis. An increasing number of studies have found that aging-related physiologic changes also affect cartilage tissues and promote the development of osteoarthritis. ${ }^{39}$ Because of the important role of mitochondrial dysfunction and oxidative stress in aging, HDAC6 may also serve as a reliable target to participate in the regulation of mitochondrial homeostasis in osteoarthritis induced by aging.

Nevertheless, HDAC6 not only promotes tubulin deacetylation but also participates in the ubiquitin protease system. Whether the effect of HDAC6 on chondrocytes in the context of osteoarthritis depends only on its deacetylase activity has not been confirmed here. Besides, how HDAC6 improves the spatial positioning of mitochondria remains unknown. Because the inhibition of HDAC6 activity has been reported to promote the occurrence of autophagic flux, ${ }^{40}$ this inhibition may promote mitophagy to recycle damaged mitochondria. The inhibition of HDAC6 may also directly act on the mitochondrial biogenesis signal pathway to promote mitochondrial production. ${ }^{31}$ Collectively, this study indicates that the expression of HDAC6 is enhanced in chondrocytes during the osteoarthritis process, and the up-regulation of HDAC6 is correlated with the abnormal distribution of mitochondria, whereas inhibition of HDAC6 activity by Tubastatin A improves the arrangement of mitochondria to improve mitochondrial function through acetylating $\alpha$-tubulin and increasing the number of mitochondria, which may contribute to inhibiting degradation of the ECM and postponing the progression of osteoarthritis (Figure 7). Therefore, HDAC6 is defined as a regulator of mitochondrial function and a potential target for treatment of osteoarthritis and supports the administration of Tubastatin A to treat osteoarthritis.

\section{Acknowledgment}

We thank the colleagues in Dr. Gao's laboratory who are not listed as coauthors.

\section{References}

1. Jones IA, Togashi R, Wilson ML, Heckmann N, Vangsness CT Jr: Intra-articular treatment options for knee osteoarthritis. Nat Rev Rheumatol 2019, 15:77-90

2. Nelson AE: Osteoarthritis year in review 2017: clinical. Osteoarthritis Cartilage 2018, 26:319-325 
3. Hunter DJ, Bierma-Zeinstra S: Osteoarthritis. Lancet 2019, 393: $1745-1759$

4. Jay GD, Elsaid KA, Kelly KA, Anderson SC, Zhang L, Teeple E, Waller K, Fleming BC: Prevention of cartilage degeneration and gait asymmetry by lubricin tribosupplementation in the rat following anterior cruciate ligament transection. Arthritis Rheum 2012, 64: $1162-1171$

5. Goutas A, Syrrou C, Papathanasiou I, Tsezou A, Trachana V: The autophagic response to oxidative stress in osteoarthritic chondrocytes is deregulated. Free Radic Biol Med 2018, 126:122-132

6. Khan NM, Ahmad I, Haqqi TM: Nrf2/ARE pathway attenuates oxidative and apoptotic response in human osteoarthritis chondrocytes by activating ERK1/2/ELK1-P70S6K-P90RSK signaling axis. Free Radic Biol Med 2018, 116:159-171

7. Li YS, Xiao WF, Luo W: Cellular aging towards osteoarthritis. Mech Ageing Dev 2017, 162:80-84

8. Benjamin M, Archer CW, Ralphs JR: Cytoskeleton of cartilage cells. Microsc Res Tech 1994, 28:372-377

9. Diegelmann RF, Peterkofsky B: Inhibition of collagen secretion from bone and cultured fibroblasts by microtubular disruptive drugs. Proc Natl Acad Sci U S A 1972, 69:892-896

10. Bauer EA, Valle KJ: Colchicine-induced modulation of collagenase in human skin fibroblast cultures, I; stimulation of enzyme synthesis in normal cells. J Invest Dermatol 1982, 79:398-402

11. Harris ED Jr, Krane SM: Effects of colchicine on collagenase in cultures of rheumatoid synovium. Arthritis Rheum 1971, 14:669-684

12. Jansen HW, Bornstein P: Effects of antimicrotubular agents on glycosaminoglycan synthesis and secretion by embryonic chick cartilage and chondrocytes. Biochim Biophys Acta 1974, 362: 150-159

13. Bodo M, Carinci P, Baroni T, Becchetti E, Bellucci C, Pezzetti F, Giammarioli M, Stabellini G, Arena N: Collagen synthesis and cell growth in chick embryo fibroblasts: influence of colchicine, cytochalasin B and concanavalin A. Cell Biol. Int 1996, 20:177-185

14. Janke C, Bulinski JC: Post-translational regulation of the microtubule cytoskeleton: mechanisms and functions. Nat Rev Mol Cell Biol 2011, 12:773-786

15. Bigman LS, Levy Y: Tubulin tails and their modifications regulate protein diffusion on microtubules. Proc Natl Acad Sci U S A 2020, $117: 8876-8883$

16. Hubbert C, Guardiola A, Shao R, Kawaguchi Y, Ito A, Nixon A, Yoshida M, Wang XF, Yao TP: HDAC6 is a microtubule-associated deacetylase. Nature 2002, 417:455-458

17. Zheng Z, Zhou Y, Ye L, Lu Q, Zhang K, Zhang J, Xie L, Wu Y, Xu K, Zhang H, Xiao J: Histone deacetylase 6 inhibition restores autophagic flux to promote functional recovery after spinal cord injury. Exp Neurol 2020, 324:113138

18. Youn GS, Lee KW, Choi SY, Park J: Overexpression of HDAC6 induces pro-inflammatory responses by regulating ROS-MAPK-NFkappaB/AP-1 signaling pathways in macrophages. Free Radic Biol Med 2016, 97:14-23

19. Wang J, Zhao L, Wei Z, Zhang X, Wang Y, Li F, Fu Y, Liu B: Inhibition of histone deacetylase reduces lipopolysaccharide-inducedinflammation in primary mammary epithelial cells by regulating ROS-NF-small ka, CyrillicB signaling pathways. Int Immunopharmacol 2018, 56:230-234

20. Li L, Liu F, Huang W, Wang J, Wan Y, Li M, Pang Y, Yin Z: Ricolinostat (ACY-1215) inhibits VEGF expression via PI3K/AKT pathway and promotes apoptosis in osteoarthritic osteoblasts. Biomed Pharmacother 2019, 118:109357

21. Cheng C, Shan W, Huang W, Ding Z, Cui G, Liu F, Lu W, Xu J, He W, Yin Z: ACY-1215 exhibits anti-inflammatory and chondroprotective effects in human osteoarthritis chondrocytes via inhibition of STAT3 and NF-kappaB signaling pathways. Biomed Pharmacother 2019, 109:2464-2471
22. Committee for the Update of the Guide for the Care and Use of Laboratory Animals; National Research Council: Guide for the Care and Use of Laboratory Animals: Eighth Edition. Washington, DC, National Academies Press, 2011

23. Glasson SS, Blanchet TJ, Morris EA: The surgical destabilization of the medial meniscus (DMM) model of osteoarthritis in the 129/SvEv mouse. Osteoarthritis Cartilage 2007, 15:1061-1069

24. Ferreira-Gomes J, Adaes S, Castro-Lopes JM: Assessment of movement-evoked pain in osteoarthritis by the knee-bend and CatWalk tests: a clinically relevant study. J Pain 2008, 9:945-954

25. Lewis JS, Hembree WC, Furman BD, Tippets L, Cattel D, Huebner JL, Little D, DeFrate LE, Kraus VB, Guilak F, Olson SA: Acute joint pathology and synovial inflammation is associated with increased intra-articular fracture severity in the mouse knee. Osteoarthritis Cartilage 2011, 19:864-873

26. Butler KV, Kalin J, Brochier C, Vistoli G, Langley B, Kozikowski AP: Rational design and simple chemistry yield a superior, neuroprotective HDAC6 inhibitor, tubastatin A. J Am Chem Soc 2010, 132:10842-10846

27. Boyault C, Sadoul K, Pabion M, Khochbin S: HDAC6, at the crossroads between cytoskeleton and cell signaling by acetylation and ubiquitination. Oncogene 2007, 26:5468-5476

28. Misawa T, Takahama M, Kozaki T, Lee H, Zou J, Saitoh T, Akira S: Microtubule-driven spatial arrangement of mitochondria promotes activation of the NLRP3 inflammasome. Nat Immunol 2013, 14. $454-460$

29. Friedman JR, Webster BM, Mastronarde DN, Verhey KJ, Voeltz GK: ER sliding dynamics and ER-mitochondrial contacts occur on acetylated microtubules. J Cell Biol 2010, 190:363-375

30. Lee JY, Nagano Y, Taylor JP, Lim KL, Yao TP: Disease-causing mutations in parkin impair mitochondrial ubiquitination, aggregation, and HDAC6-dependent mitophagy. J Cell Biol 2010, 189: 671-679

31. Chen J, Shi X, Padmanabhan R, Wang Q, Wu Z, Stevenson SC, Hild M, Garza D, Li H: Identification of novel modulators of mitochondrial function by a genome-wide RNAi screen in Drosophila melanogaster. Genome Res 2008, 18:123-136

32. Cerveny KL, Tamura Y, Zhang Z, Jensen RE, Sesaki H: Regulation of mitochondrial fusion and division. Trends Cell Biol 2007, 17:563-569

33. Maneiro E, Martin MA, de Andres MC, Lopez-Armada MJ, Fernandez-Sueiro JL, del Hoyo P, Galdo F, Arenas J, Blanco FJ: Mitochondrial respiratory activity is altered in osteoarthritic human articular chondrocytes. Arthritis Rheum 2003, 48:700-708

34. Blanco FJ, Rego I, Ruiz-Romero C: The role of mitochondria in osteoarthritis. Nat Rev Rheumatol 2011, 7:161-169

35. Shao X, Chen Q, Dou X, Chen L, Wu J, Zhang W, Shao H, Ling P, Liu F, Wang F: Lower range of molecular weight of xanthan gum inhibits cartilage matrix destruction via intrinsic bax-mitochondria cytochrome c-caspase pathway. Carbohydr Polym 2018, 198: 354-363

36. Kim HA, Blanco FJ: Cell death and apoptosis in osteoarthritic cartilage. Curr Drug Targets 2007, 8:333-345

37. Que Q, Guo X, Zhan L, Chen S, Zhang Z, Ni X, Ye B, Wan S: The GLP-1 agonist, liraglutide, ameliorates inflammation through the activation of the PKA/CREB pathway in a rat model of knee osteoarthritis. J Inflamm (Lond) 2019, 16:13

38. Shah R, Raska K Jr, Tiku ML: The presence of molecular markers of in vivo lipid peroxidation in osteoarthritic cartilage: a pathogenic role in osteoarthritis. Arthritis Rheum 2005, 52:2799-2807

39. Loeser RF, Collins JA, Diekman BO: Ageing and the pathogenesis of osteoarthritis. Nat Rev Rheumatol 2016, 12:412-420

40. Majora M, Sondenheimer K, Knechten M, Uthe I, Esser C, Schiavi A, Ventura N, Krutmann J: HDAC inhibition improves autophagic and lysosomal function to prevent loss of subcutaneous fat in a mouse model of Cockayne syndrome. Sci Transl Med 2018, 10 